\title{
Low Cost Neutron and Muon Detectors for Soil Moisture Monitoring
}

\section{P. Stowell, ${ }^{a, c, *}$ A. M. Brown, ${ }^{a}$ P. Chadwick, ${ }^{a}$ S. Fargher,${ }^{b}$ C. Rulten, ${ }^{a}$ C. Steer ${ }^{c}$ and L. F. Thompson ${ }^{b, c}$}

${ }^{a}$ Centre for Advanced Instrumentation, Durham University, South Road, Durham, DH1 3LE, UK

${ }^{b}$ Physics \& Astronomy Department, University of Sheffield, Hicks Building, S3 7RH

${ }^{c}$ Geoptic Infrastructure Investigations Ltd, Beechcroft Barn, Pickaxe Lane, South Warnborough, RG29 IS

E-mail: john.p.stowell@durham.ac.uk

Water scarcity is a significant challenge for the world's population. With the likelihood of extreme droughts increasing each year, technologies to promote sustainable irrigation and improve resilience to water shortage are needed. Continuous monitoring of soil moisture in arid regions is a major problem as existing techniques such as point sensors or satellite mapping can have high associated costs per hectare. Cosmic Ray Neutron Sensing (CRNS) of soil moisture is a well established technique in the hydrological community. Helium-3 CRNS probes placed above a site can detect cosmic ray neutrons backscattered from the surrounding soil 130-240m away. By monitoring the variation in the total neutrons observed over time (and correcting for cosmic ray intensity) it is possible to estimate the average volumetric soil moisture content for a site. With a large detector footprint, the technique can bridge the difference in length scales between point probes and satellite data, however the high cost of Helium-3 is a barrier for adoption outside of the hydrological research community. In this work we present ongoing efforts to develop new lithium and boron based cosmic ray detectors as alternatives to expensive Helium-3 detectors. Taking advantage of developments in scintillator composites within the nuclear industry, and low power single photon counting instrumentation, these cost efficient detectors will be specifically optimised for use on smallholder farms. The optimisation and testing of these new systems is presented before discussing the use of low cost muon sensors to automatically correct for temporal variations in the incoming cosmic ray intensity.

$37^{\text {th }}$ International Cosmic Ray Conference (ICRC 2021)

July 12 th - 23rd, 2021

Online - Berlin, Germany

\footnotetext{
${ }^{*}$ Presenter
} 


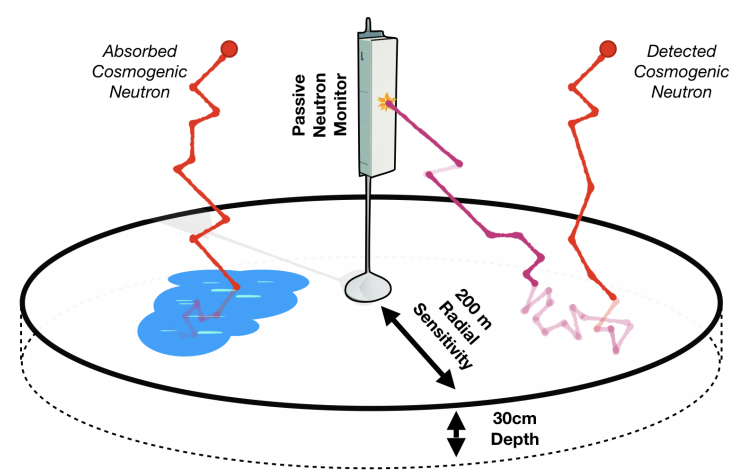

Figure 1: Summary of the Cosmic Ray Neutron Sensing (CRNS) technique. Since cosmogenic neutrons are more likely to be absorbed in the soil when hydrogen content is high, the number of neutrons detected by a passive neutron monitor placed above ground is inversely correlated with the local hydrogen content in a $200 \mathrm{~m}$ radial area, and down to approximately $30 \mathrm{~cm}$ soil depth.

\section{Introduction}

Continuous monitoring of soil moisture in arid regions is a major problem as existing techniques can have high associated costs per hectare. Whilst in ground sensors can provide relatively low cost soil moisture measurements at a single point, extrapolating their readings to a large site requires good knowledge of how variations in local soil composition can modify sensitivity. Satellite data has been shown to provide a competitive cost/hectare when mapping large sites, however the resolution is typically at the kilometre scale [1], and continuous measurements at high temporal resolution, like those needed to support smart irrigation plans, can be prohibitively expensive. Cosmic rays offer a unique solution to the problem of measuring soil moisture at the field scale for hydrological monitoring applications. The Cosmic Ray Neutron Sensing (CRNS) method is an alternative sensing technique that can be used to non-invasively monitor soil Volumetric Water Content (VWC) [2]. Since neutrons have a high interaction probability with hydrogen, the number of cosmic ray neutrons back scattered from a soil is known to be inversely correlated with the soil's hydrogen content.

As shown in Figure 1, a neutron detector placed above ground is sensitive to variations in the local water content due to an increase in soil hydrogen moderating the neutrons such that they are more likely to be absorbed before they leave the soil. The correlation between neutron rate and local VWC is strongest in the epithermal energy range, between $1 \mathrm{eV}$ to $1 \mathrm{MeV}$. Given a neutron count rate measurement in this region of interest, $N$, the variation in VWC is typically estimated as

$$
V W C \sim\left(\frac{0.0869}{f_{p} f_{c} \frac{N}{N_{0}}-0.3720}-0.1236\right),
$$

where $N_{0}$ is the expected counting rate for dry soils, and $f_{p}$ and $f_{c}$ are correction factors for local environmental conditions (pressure, absolute humidity, soil composition), and varying incoming cosmic ray intensity. Given the large path length of neutrons in air within this energy range, a neutron detector can be sensitive to variations in soil moisture content up to $200 \mathrm{~m}$ away. Because of variations in average penetration depth due to soil moisture and air humidity, the actual sensitive 
footprint is dependent on local conditions. Studies in [3] have found that this range is typically between 130-240m depending on soil conditions.

A large number of CRNS stations have been deployed around the world in the past ten years for hydrological monitoring purposes. Typically these have employed helium-3 (He-3) or borontrifluoride (BF3) based systems to detect neutrons in the epithermal range. Whilst extremely effective, the adoption of this technology outside of academic research groups within agriculture has been relatively slow. This is a result of the high inherent cost of He-3 based neutron detectors, and the safety implications surrounding the toxicity of BF3. For the CRNS technique to be a viable solution for hydrological monitoring in agriculture, lower cost and safer detector systems are needed. Already a number of groups have developed CRNS specific alternatives based on Boron-10 lined proportional tubes [4] or composite scintillator detectors [5] to overcome these challenges.

In these proceedings we review efforts to develop a low cost scintillating cosmic ray neutron detector system for agricultural applications where cost may be a bigger driving factor than temporal resolution. The work is organised as follows: Section 2 discusses the development of the first iteration of these systems which are based on lithium-6 loaded scintillator foils, Section 3 discusses ongoing work to produce lower cost boron loaded scintillator detectors, Section 4 discusses the feasibility of using cosmic ray muons to monitor local cosmic ray intensity, before Section 5 discusses the next steps to realise a low cost scintillating CRNS detector solution for the agricultural sector.

\section{2. $\mathbf{L i}^{6}$ FZnS Detector Prototyping}

The use of Lithium- $6\left({ }^{6} \mathrm{Li}\right)$ loaded scintillator foils is a well established technique for production of large area thermal neutron detectors [6]. When exposed to thermal neutrons, ${ }^{6} \mathrm{Li}$ has a high probability of absorbing a neutron before subsequently decaying into an alpha and triton with a combined Q-value of $4.8 \mathrm{MeV}$. When uniformly mixed with a bright scintillating phosphor such as Zinc-Sulphide $(\mathrm{ZnS})$ it is possible to detect the positive charged byproducts of this neutron induced nuclear decay. When this scintillating composite is manufactured into thin foils significantly less than the typical radiation length of $\mathrm{ZnS}$, it is also possible to make effectively 'gamma-blind' detectors that can discriminate background gamma interactions from neutrons based on the overall amplitude and width of the resultant scintillator pulse.

Several companies worldwide now offer neutron portal monitor products to the nuclear safeguarding community (for example [7]), and as a result, standardized thermal neutron scintillator screens can now be purchased as a commercial off the shelf product as an alternative to gaseous neutron detector technologies (e.g. EJ-426 from Eljen [8]) The technical requirements for portal monitoring applications are similar to that of the CRNS technique, namely a relatively low cost per unit area, and the ability to discriminate between neutrons and other electromagnetic radiation induced backgrounds. To minimize cost, these systems typically employ large sheets of scintillator such as EJ-426 and wavelength shifting paddles to allow a single photo-multiplier to be effectively coupled to a much larger area of scintillating material. Recently scintillating neutron detectors which combined both a fast neutron detector (EJ-299-33 [9]) and thermal neutron detector (EJ-426) were found to achieve an efficiency of approximately 55\% that of a traditional CRNS He-3 based system, despite a significantly lower cost [5]. The now widespread availability of components 


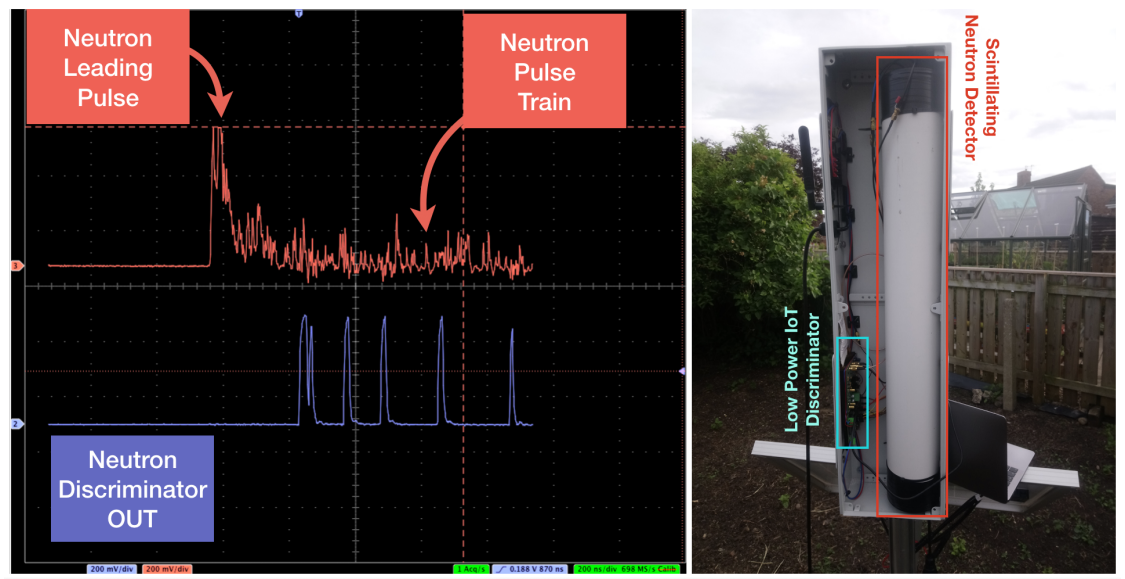

Figure 2: ${ }^{6} \mathrm{LiFZnS} \mathrm{CRNS} \mathrm{detector} \mathrm{prototype} \mathrm{system.} \mathrm{(Left)} \mathrm{Example} \mathrm{amplified} \mathrm{photo-multiplier} \mathrm{pulse}$ obtained from a neutron induced event, and subsequent FPGA generated neutron trigger signals. (Right) Field ready integrated CRNS system, showing the relative size of the neutron detector tube and data acquisition system.

required for these systems, makes scintillator based alternatives to Helium-3 detectors a promising avenue of research to explore when attempting to reduce the cost of the CRNS technique.

In this work, we follow a slightly different approach to that shown in [5], investigating the feasibility of assembling low cost neutron detectors out of only a single sheet of ${ }^{6} \mathrm{LiFZnS}$ thermal neutron scintillator. These detectors, discussed in detail in [10], are closer in construction to typical scintillator based neutron portal monitors, but consist of a cylindrical wavelength shifting light guide wrapped in a ${ }^{6} \mathrm{LiFZnS}$ thermal neutron scintillator material. Maintaining a cylindrical shape means light guides can be constructed from low cost wavelength shifting rods, and allows for efficient coupling of a single $38 \mathrm{~mm}$ diameter Photo-Multiplier Tube (PMT) to a $600 \mathrm{~cm}^{2}$ area of scintillator. Given the long decay time of ${ }^{6} \mathrm{LiFZnS}$ scintillator foils, and low sensitivity to gamma radiation, it is possible to discriminate neutron induced events from background based purely on the width of the resultant PMT pulse above a fixed threshold. This approach allows detectors to be constructed that do not require fast pulse shape digitization for efficient particle discrimination, reducing overall power consumption and cost.

A low power time-over-threshold discriminator has been developed based on a similar trigger system described in [12]. Signals from the PMT are amplified by a fast front end amplifier stage before being split into two separate fixed threshold discriminators. The signals from these discriminators are routed to a Field Programmable Gate Array (FPGA) to allow the time over threshold of the PMT signals to be continuously evaluated in real time with a $5 \mathrm{~ns}$ resolution. The threshold of one discriminator is optimised to trigger when the PMT signal passes above a high threshold to capture the leading pulse of a large scintillation event. This leading edge trigger subsequently starts the counting of secondary lower amplitude discriminator triggers. The threshold for this stage is optimised to count the number of secondary pulses occurring in the subsequent decaying scintillator 'pulse train' that follows a neutron induced event. A multi-stage filter algorithm is used to count the number of coincident pulses in this train within a given window and issue a neutron discriminator trigger when it reaches a programmable threshold. As shown in Figure 2, 

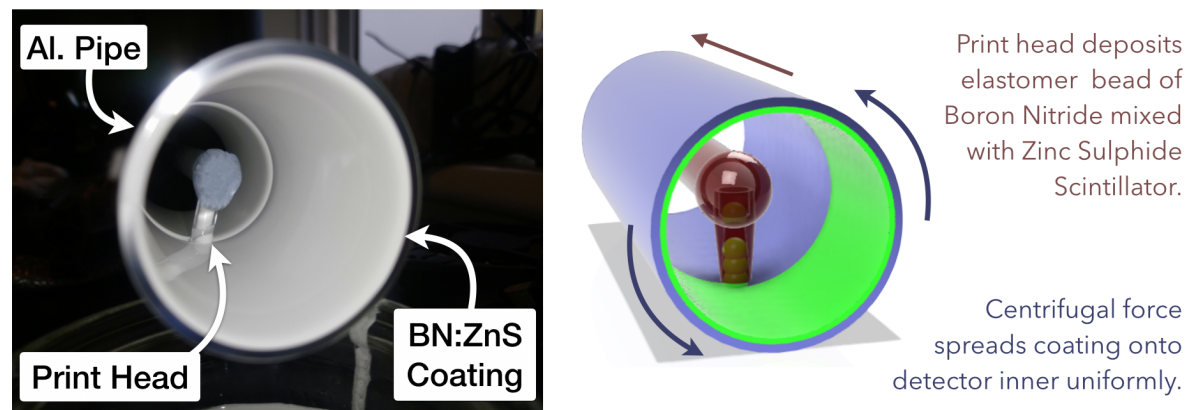

Figure 3: Spin coating technique. (Left) example of an aluminium pipe being coated in a thin layer of scintillating material. (Right) Explanation of the spin coating procedure.

multiple neutron triggers can be issued in each event as the scintillator decays, but only the first trigger signal in any $100 \mu$ s hold-off period is used to count the number of neutron interactions and update estimates of incoming neutron flux.

A soft SPI core is included in the FPGA firmware to allow the entire neutron detection unit to interface directly with a range of external micro-controller master devices. Included in this interface are digitally programmable thresholds and PMT high voltage bias control. This FPGA system is controlled by an ESP32 based GSM module which can transmit data directly to an open source Internet-Of-Things database for further offline processing and monitoring. Initial tests of this integrated system has shown a good sensitivity to neutrons in the epithermal range. The entire detector system (PMT, High Voltage, Discriminator, FPGA, ESP32) has a power consumption less than $1.5 \mathrm{~W}$, allowing it to be powered by a compact solar panel. This makes it a portable and autonomous neutron monitoring solution suitable for field deployment. Three of these integrated monitoring systems have recently been deployed in the UK to characterize the overall system response to varying soil moisture and understand the data acquisition system's stability under different environmental parameters.

\section{BNZnS Scintillator Development}

${ }^{6} \mathrm{LiFZnS}$ based alternatives to Helium-3 detectors represent a promising first step in reducing the overall cost of CRNS systems, however even lower cost detection technologies are still needed for the technique to be truly viable for smallholder farm use. Since Lithium- 6 is a controlled nuclear substance the cost is still relatively large when compared to other industrial soil moisture sensors. In addition, Lithium- 6 comes with additional import/export restrictions that could limit widespread global adoption in the future.

Un-enriched Boron Nitride (BN) is one possible alternative to Lithium-6 that could be used to produce ultra low cost neutron detectors at-scale [11]. Natural $\mathrm{BN}$ is non-toxic, readily available for pharmaceutical applications, and contains $20 \%{ }^{10} \mathrm{~B}$ which has a high neutron absorption probability. Large area BNZnS detectors are therefore capable of achieving similar sensitivities to ${ }^{6} \mathrm{LiFZnS}$ composites provided efficient light collection methods can be used to overcome the reduced Q-value of the ${ }^{10} \mathrm{~B}+n$ reaction when compared to ${ }^{6} \mathrm{Li}+n(2.3 \mathrm{MeV}$ compared to $4.8 \mathrm{MeV})$. 

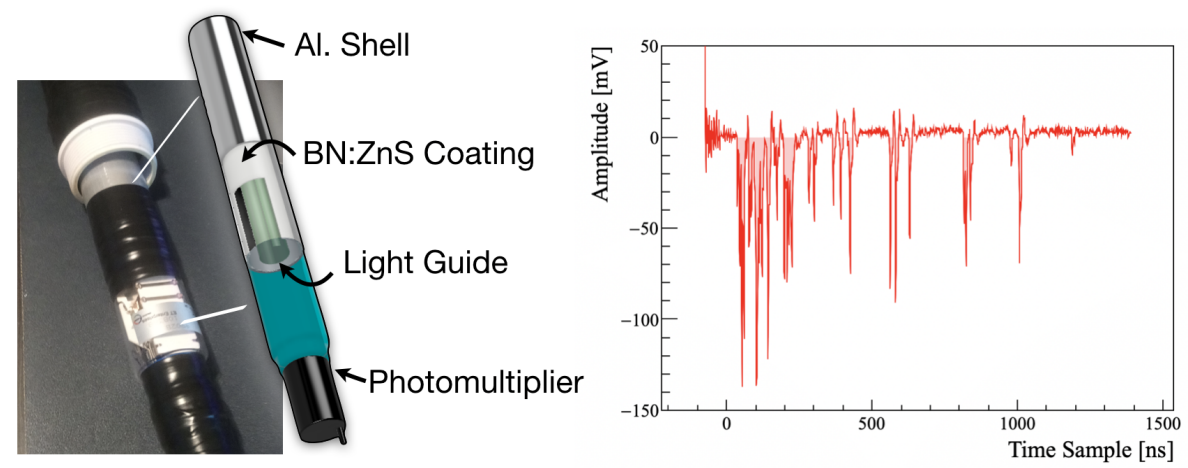

Figure 4: First BNZnS spin coated deetector prototype tests. (Left) detector structure of the next iteration of the scintillating neutron detectors. (Right) Example neutron pulse train obtained when this prototype was exposed to a Cf-252 neutron radiation source.

We are currently developing new BNZnS based cosmic ray detector alternatives as the next iteration of the ${ }^{6} \mathrm{LiFZnS}$ based detectors discussed in the previous section. One disadvantage of Boron Nitride is the lack of 'commercial off the shelf' solutions for BNZnS composite scintillators. To overcome this challenge, a detector coating procedure has been developed (referred to as 'rotary spin coating' throughout), to deposit scintillator composites onto the inside of light tight aluminium detector enclosures. As shown in Figure 3 the rotary spin coating procedure involves mixing Boron Nitride and Zinc Sulphide into a fast setting polymer before depositing it by moving a bead of solution across a surface using a remotely controlled print head. This print head is moved along the inside of an aluminium pipe which is rotated at speed as scintillating mixture is deposited so that the centrifugal force spreads the mixture into a uniform coating. The thickness of this coating can be determined by the speed the print head moves, and the volume of solution deposited. Depositing scintillator directly onto a light tight aluminium enclosure using the spin coating technique allows wavelength shifting light guides and PMTs to be be inserted directly into the pipe after printing, simplifying the overall build process and reducing cost. Initial tests of spin coated detectors, like that shown in Figure 4, have found good sensitivity to neutron radiation sources. Work is now underway to scale up this production method to support further detector comparisons against ${ }^{6} \mathrm{LiFZnS}$ based systems.

\section{Muon Monitoring}

As shown in Equation 1, the CRNS technique requires variations in the local direct cosmic ray intensity to be corrected for $\left(f_{c}\right)$ before the volumetric water content can be extracted. Typically data from the Jungfraujoch neutron monitors is used to correct for these variations by requesting data directly from the worldwide neutron monitor database [13]. Whilst suitable for most applications, this approach is not viable for sites were continuous monitoring is required but wide area internet access is unreliable. One alternative approach is the monitoring of local changes in the electromagnetic component of low energy cosmic ray showers.

It is possible to detect muons with relatively low cost electronics, therefore a muon tracker is likely to offer a cost-effective way to measure these variations independent of local soil moisture 


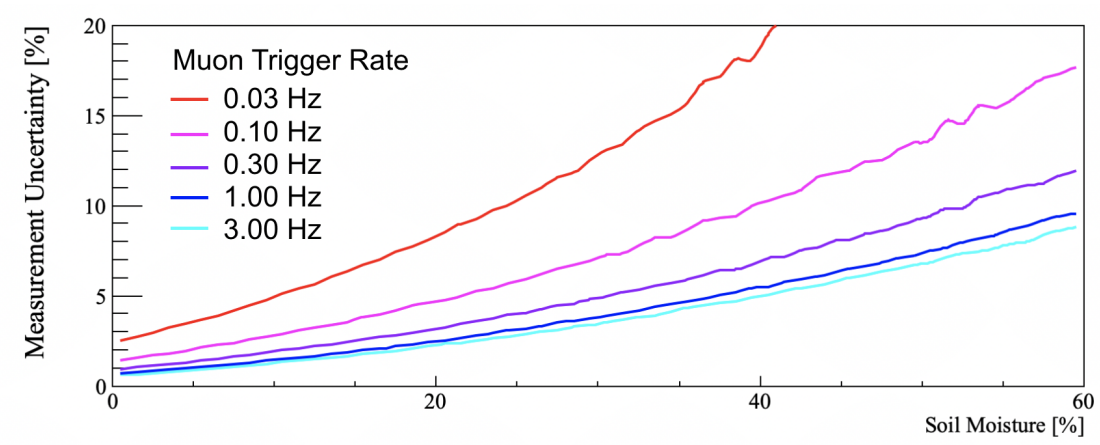

Figure 5: Expected measurement statistical uncertainty when using local muon counting rates to correct cosmic ray intensity. All uncertainty estimates assume an hour long integration time.

variations. As shown in Figure 5, for an alternative method of calculating $f_{c}$ to be effective it must have a statistical uncertainty less than that of a typical neutron detector itself (assumed to be 2000 counts per hour), otherwise the measurement uncertainty can be significant when the soil moisture is high. We have found that a muon detector system needed to achieve a suitable statistical uncertainty of less than $1 \%$ after 1 hour for dry soils is approximately $400 \mathrm{~cm}^{2}$ (equivalent to two $20 \mathrm{~cm} \times 20 \mathrm{~cm}$ scintillator panels spaced $5 \mathrm{~cm}$ apart). This is small enough that it could be easily installed in off the shelf weatherproof enclosures for field deployment. Importantly, the proposed muon tracking system size is only suitable for typical 1 hour resolution cosmic ray sensing deployments, and in cases where improved timing resolution is needed larger area muon trackers would be required to ensure that statistical uncertainty of the cosmic ray intensity is not the dominant uncertainty in Equation 1. Since the temporal variation in cosmic ray intensity is expected to remain approximately uniform across a single farm site it is possible that a single muon system of this size could support the extraction of VWC from multiple nearby neutron detector systems provided that local networking capabilities such as LoRaWAN are available.

\section{Discussion and Outlook}

The detectors shown here represent a promising first step in the development of ultra low cost neutron detectors for hydrological monitoring on smallholder farms. Using thermal neutron scintillator foils as the primary detector component without additional fast scintillator minimises the detectors sensitivity to background gamma radiation, allowing low power dual threshold discriminator circuits to be used to identify neutrons. Three test systems have recently been deployed at farm sites across the UK to characterise the response of these systems to long term environmental effects. Work is ongoing to develop a small scale muon tracker that could be integrated with these IoT enabled neutron sensors to remove the need to obtain data from the worldwide neutron monitor database and support further adoption of the CRNS sensing technique in remote areas.

\section{Acknowledgements}

P. Stowell would like to thank the Royal Commission for the Exhibition of 1851 for his research fellowship that has supported the majority of this work. The authors would also like to thank Julia 
Cooper, and James Standen at Newcastle University, and Richard Grayson, Marcelo Galdos, and Murilo Vianna at Leeeds university for their support in deploying early neutron detector prototypes. The authors would also like to support the STFC Food Network+ for their support in the production of two of the first ${ }^{6} \mathrm{LiFZnS}$ detector systems. We acknowledge the NMDB database (www.nmdb.eu), founded under the European Union's FP7 programme (contract no. 213007) for providing data used to test early neutron detector prototypes.

\section{References}

[1] Cui, C., et. Al (2018). Soil moisture mapping from satellites: An intercomparison of SMAP, SMOS, FY3B, AMSR2, and ESA CCI over two dense network regions at different spatial scales. Remote Sensing, 10(1), 33.

[2] Franz, T. E., et. Al (2012). Field validation of a cosmic-ray neutron sensor using a distributed sensor network. Vadose Zone Journal, 11(4), vzj2012-0046.

[3] Köhli, M., et. Al (2015). Footprint characteristics revised for field-scale soil moisture monitoring with cosmic-ray neutrons. Water Resources Research, 51(7), 5772-5790.

[4] Weimar, Jannis, et al. "Large-scale boron-lined neutron detection systems as a $3 \mathrm{He}$ alternative for cosmic ray neutron sensing." Frontiers in Water 2 (2020): 16.

[5] Stevanato, Luca, et al. "A novel cosmic-ray neutron sensor for soil moisture estimation over large areas." Agriculture 9.9 (2019): 202.

[6] Barton, John C., Christopher J. Hatton, and John E. McMillan. "A novel neutron multiplicity detector using lithium fluoride and zinc sulphide scintillator." Journal of Physics G: Nuclear and Particle Physics 17.12 (1991): 1885.

[7] Symetric Product Webpage, "https://symetrica.com/technology/"

[8] Eljen EJ-426 Product Webpage " https://eljentechnology.com/products/neutron-detectors/ej$42 "$

[9] Eljen EJ-299-33 Product Webpage "https://eljentechnology.com/products/plasticscintillators/ej-299-33a-ej-299-34"

[10] Stowell, J. P., et al. "Optimising Scintillating Neutron Detectors for Cosmic Ray Soil Moisture Monitoring." arXiv preprint arXiv:2106.06757 (2021).

[11] Marsden, Edward James. Large area thermal neutron detectors for security applications. Diss. University of Sheffield, 2013.

[12] Stoykov, A., et al. "A SiPM-based ZnS: 6LiF scintillation neutron detector." Nuclear Instruments and Methods in Physics Research Section A: Accelerators, Spectrometers, Detectors and Associated Equipment 787 (2015): 361-366.

[13] Worlwide Neutron Monitoring Database, "https://www.nmdb.eu" 